\title{
Applicability of the High Field Model: A Preliminary Numerical Study
}

\author{
CARLO CERCIGNANI ${ }^{a}$, IRENE M. GAMBA ${ }^{\mathrm{b}}$, JOSEPH W. JEROME ${ }^{\mathrm{c}, *}$ and CHI-WANG SHU ${ }^{\mathrm{d}}$ \\ ${ }^{\mathrm{a}}$ Politecnico di Milano, 20133 Milano, Italy; ${ }^{\mathrm{b}}$ Courant Institute, New York University, New York, NY 10012; \\ ${ }^{\mathrm{c}}$ Department of Mathematics, Northwestern University, Evanston, IL 60208; \\ ${ }^{\mathrm{d}}$ Division of Applied Mathematics, Brown University, Providence, RI 02912
}

\begin{abstract}
In a companion presentation, we have discussed the theory of a mesoscopic/ macroscopic model, which can be viewed as an augmented drift-diffusion model. Here, we describe how that model is used. The device we consider for this presentation is the one dimensional GaAs $n^{+}-n-n^{+}$structure of length $0.8 \mu \mathrm{m}$. First, a full HydroDynamic (HD) model, proven reliable when compared with Monte Carlo simulations, is used to simulate the device via the ENO finite difference method. As applied to the full device, the new model is not necessarily superior to traditional Drift-Diffusion (DD). Indeed, when we plot the quantity $\eta=\mu_{0} E / \sqrt{k T_{0} / m}$, where $\mu_{0}$ is the mobility constant and $E=-\phi^{\prime}$ is the electric field, we verify that the high field assumption $\eta>1$, required for the high field model, is satisfied only in an interval given approximately by $[0.2,0.5]$. When we run both the DD model and the new high field model in this restricted interval, with boundary conditions of concentration $n$ and potential $\phi$ provided by the HD results, we demonstrate that the new model outperforms the DD model. This indicates that the high field and DD models should be used only in parts of the device, connected by a transition kinetic regime. This will be a domain decomposition issue involving interface conditions and adequate numerical methods.
\end{abstract}

Keywords: Augmented drift-diffusion, high field model, domain decomposition, ENO algorithm

\section{INTRODUCTION}

In previous work, we have demonstrated the robustness of an algorithm (ENO: Essentially Non-Oscillatory) designed for the simulation of the hydrodynamic model for semiconductors over a wide range of parameters. In [5] and [6], $n^{+}-n-n^{+}$diodes in one dimension and MESFETS in two dimensions were simulated. The present paper deals with the high field model introduced in [3], and appearing elsewhere in these proceedings [2].

\footnotetext{
*Corresponding author: Tel.: (847) 491-5575, Fax: (847) 491-8906, e-mail: jwj@math.nwu.edu.
} 


\section{THE MODELS}

The new model is an extension of the DriftDiffusion (DD) model. Hence we first describe the DD model in a context for comparison.

\subsection{Drift-Diffusion Model in One Dimension with $\mu$ Depending On $E$}

The DD model is well documented (see, for example, [4]). It is given by:

$$
n_{t}+J_{x}=0
$$

where the representation in terms of hyperbolic and viscous components is given by

$$
J=J_{\text {hyp }}+J_{\text {vis }}
$$

and

$$
\begin{aligned}
& J_{\text {hyp }}=-\mu n E, \\
& J_{\text {vis }}=-\tau(n \theta)_{x} .
\end{aligned}
$$

Here, $n$ denotes carrier concentration and $J$ denotes current defined per unit charge modulus. We have separated current components in anticipation of the high field model to follow. Also, the electric field is denoted by $E$, so that in terms of the electrostatic potential $\phi$,

$$
E=-\phi_{x}, \quad\left(\varepsilon \phi_{x}\right)_{x}=e\left(n-n_{d}\right),
$$

where we use the customary expressions, for doping $n_{d}$, dielectric $\varepsilon$, and charge modulus $e$. Set

$$
\tau=\frac{m \mu}{e}, \quad \theta=\frac{k_{b}}{m} T_{0} .
$$

$\tau$ denotes the relaxation time, $m$ denotes effective mass and ambient temperature is denoted by $T_{0}$, with $\theta$ given in energy units. Of course, $\mu$ is mobility, and we take $\mu$ to be dependent upon the electric field $E$, using the formula (2.42) in [4]:

$$
\mu(E)=2 \mu_{0} /\left[1+\sqrt{1+4\left(\mu_{0}|E| / v_{d}\right)^{2}}\right] .
$$

Here, $v_{d}$ has the interpretation of the saturation velocity and $\mu_{\theta}$ is the low field mobility. The velocity for the $\mathrm{DD}$ model is a derived quantity computed by $v=J / n$.

The device we consider for this presentation is the one dimensional GaAs $n^{+}-n-n^{+}$structure of length $0.8 \mu \mathrm{m}$. The device used is as follows: $x \in[0,0.8]$; the doping is defined by $n_{d}(x)=10^{5} /$ $\mu \mathrm{m}^{3}$ in $0 \leq x \leq 0.1$ and in $0.5 \leq x \leq 0.8$, and by $n_{d}(x)=2 \times 10^{3}$ in $0.15 \leq x \leq 0.45$, with a smooth intermediate transition. The boundary conditions are: fixed $n$ at both ends, and fixed $\phi$ at both ends (with a difference $=v$ bias). Simulations are performed for $v$ bias $=0,0.5$ and $1.0 \mathrm{~V}$, but results are shown for $v$ bias $=1.0$ only to save space. Other parameters: $m=0.065 \times 0.9109\left(10^{-30} \mathrm{Kg}\right)$, $e=0.1602\left(10^{-18} \mathrm{C}\right), k_{b}=0.138046 \times 10^{-4}\left(10^{-18}\right.$ $\mathrm{J} /$ Kelvin $), \varepsilon=13.2 \times 8.85418\left(10^{-18} \mathrm{~F} / \mu \mathrm{m}\right)$.

In the definition of $\mu$, we take $\mu_{0}=4.0 \mu \mathrm{m}^{2} / \mathrm{V} /$ ps. We consider $T_{0}=300 \mathrm{~K}$ for which $v_{d}=0.6$ $\mu \mathrm{m} / \mathrm{ps}$, which is taken to be the maximum of the velocity in the HD run with $v$ bias $=1.0$.

The results are shown in Figure 1. They do not match as well with those of the HD model (with a doping $n_{d}$ dependent $\mu$ ), as in the silicon case which is not presented here. The oscillations in the velocity near the left junction are due to the numerical differentiation of rapidly changing quantities.

\subsection{The High Field Model}

The model can be written as follows:

$$
n_{t}+J_{x}=0
$$

where the representation in terms of hyperbolic and viscous components is now given by

$$
J=J_{\text {hyp }}+J_{\text {vis }}
$$

and

$$
\begin{aligned}
& J_{\text {hyp }}=-\mu n E+\tau \mu\left(\frac{e}{\varepsilon}\right) n(-\mu n E+\omega), \\
& J_{\text {vis }}=-\tau\left[n\left(\theta+2 \mu^{2} E^{2}\right)\right]_{x}+\tau \mu E(\mu n E)_{x}
\end{aligned}
$$



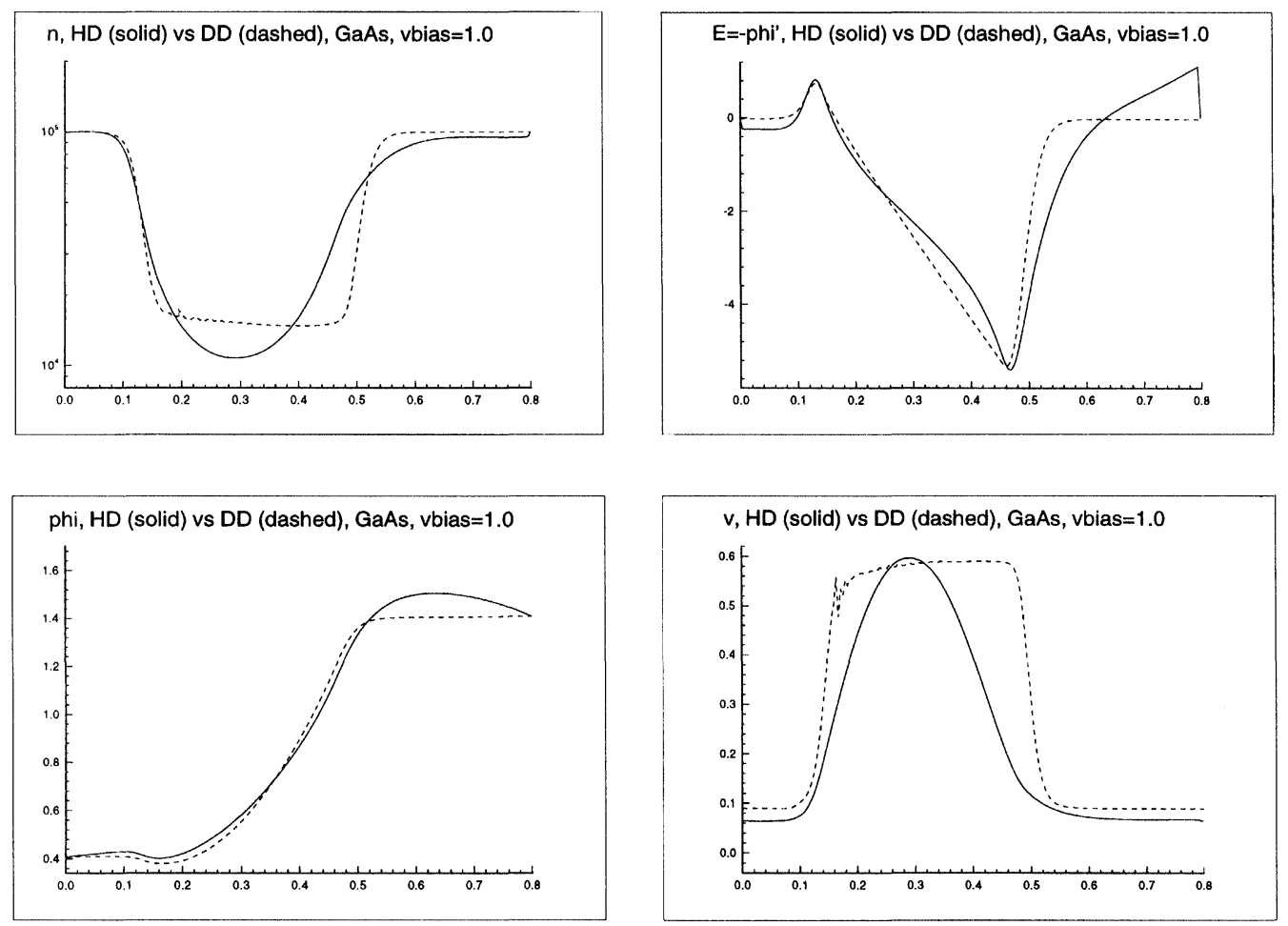

FIGURE 1 Comparison of the results of the HD model with a doping $n_{d}$ dependent $\mu$ (solid line) and that of the DD model with an electric field $E$ dependent $\mu$ (dashed line). GaAs, $T_{0}=300, v$ bias $=1.0$. Top left: concentration $n$ in $\mu \mathrm{m}^{-3}$; top right: the electric field $E=-\phi_{x}$ in volts $/ \mu \mathrm{m}$; bottom left: the potential $\phi$ in volts; bottom right: the velocity $v$ in $\mu \mathrm{m} / \mathrm{ps}$.

Also, the electrostatic equation is satisfied as in the DD model, and $\tau$ and $\theta$ are defined similarly. Furthermore, $\omega$ is taken to be a constant:

$$
\omega=\left.(\mu n E)\right|_{x=0},
$$

and the velocity is again derived quantity.

We again consider the same GaAs device as before. The results are shown in Figure 2. Not much improvement, if any, is observed over the DD results. The spikes in the velocity near the junctions are due to the derived nature of that quantity; particularly the numerical differentiation of rapidly changing quantities.

\section{RESTRICTION TO CHANNEL}

We plot the quantity $\eta=\mu_{0} E / \sqrt{\left(k T_{0} / m\right)}$ where $\mu_{0}$ is the mobility constant and $E=-\phi^{\prime}$ is the electric field (obtained from the HD simulation), in Figure 3.

We can clearly see from Figure 3 that the high field assumption $\eta>1$ is satisfied only in an interval given approximately by $[0.2,0.5]$. We thus run both the DD model and the new high field model in this restricted interval, with boundary conditions of concentration $n$ and potential $\phi$ provided by the HD results. We can see in Figure 4 that the new model decisively outperforms the DD model.

This indicates that the high field and DD models should be used only in parts of the device, connected by a transition kinetic regime. This will be a domain decomposition issue involving interface conditions and adequate numerical methods, and is currently under investigation. 

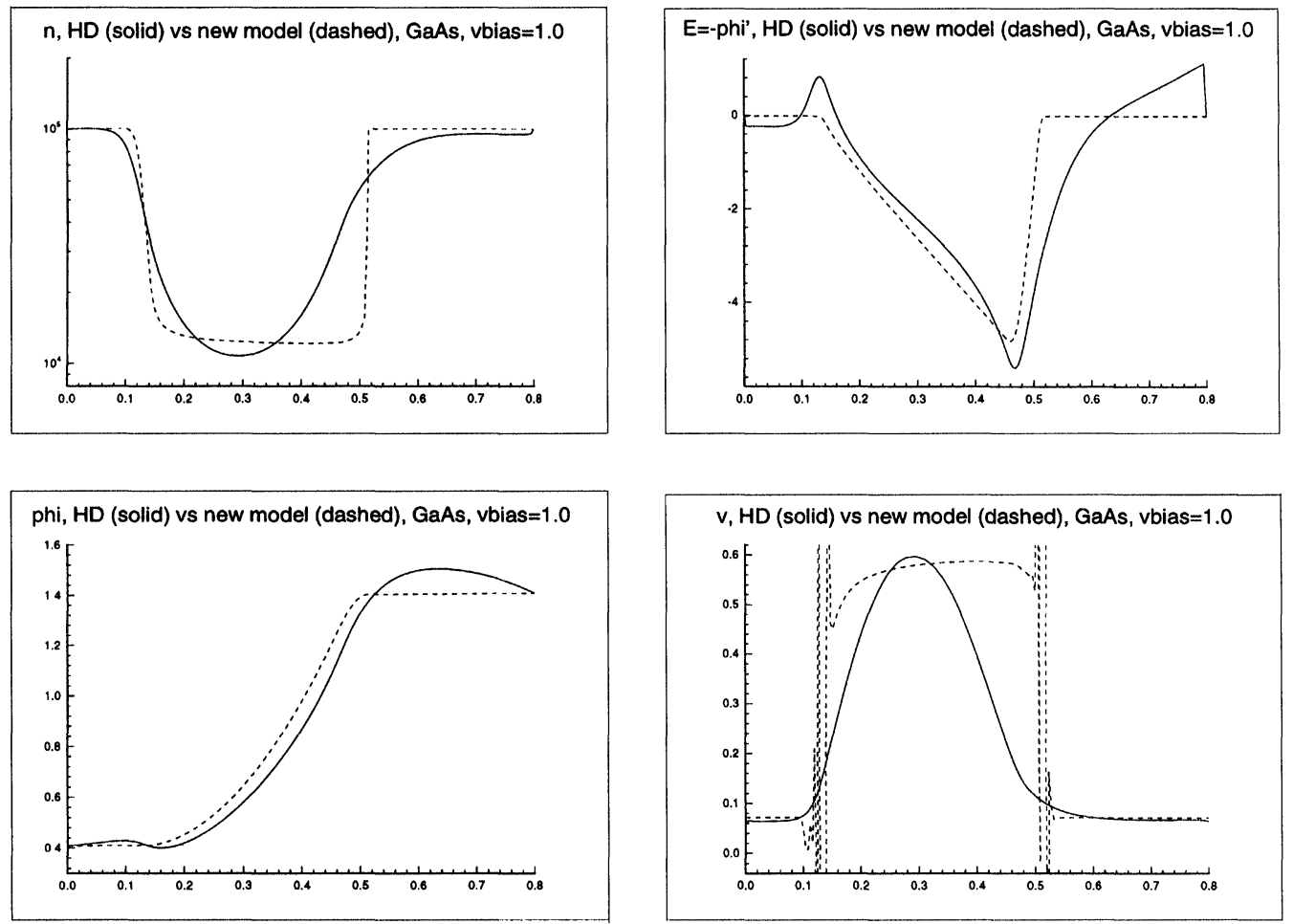

FIGURE 2 Comparison of the results of the HD model (solid line) and that of the new model (dashed line). GaAs, $T_{0}=300$, $v$ bias $=1.0$. Top left: concentration $n$ in $\mu \mathrm{m}^{-3}$; top right: the electric field $E=-\phi_{x}$ in volts $/ \mu \mathrm{m}$; bottom left: the potential $\phi$ in volts; bottom right: the velocity $v$ in $\mu \mathrm{m} / \mathrm{ps}$.

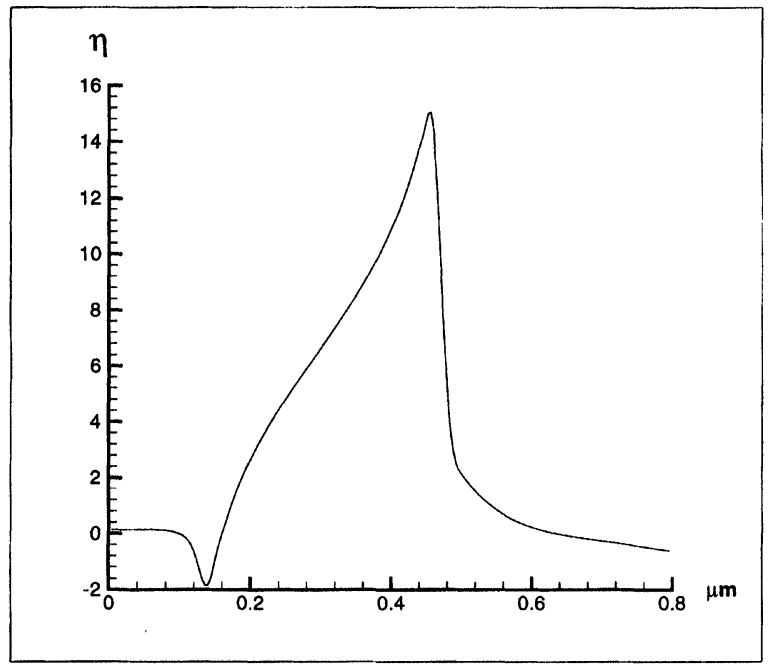

FIGURE 3 The quantity $\eta=\mu_{0} E / \sqrt{\left(k T_{0} / m\right)}$, where $E=-\phi^{\prime}$ is the electric field obtained from the HD simulation.

\section{COMPARISON WITH KINETIC AND AUGMENTED DRIFT-DIFFUSION MODELS}

The high field model may be thought of as a form of an augmented DD model. In this section, we briefly compare the results of our simulations with those of [1] and [7]. The results of [7] deal primarily with tracking the carrier drift velocity in Silicon. The model is very similar to that originally employed by Thornber [9]. We note that the perturbation terms of the high field model introduced here include more than simply differentiated electric field terms. Comparisons are made in [7] among various models, including DD and augmented DD, as well as the hydrodynamic model and Monte-Carlo simulation. In these studies, the authors of [7] tended to find that standard DD understated drift velocity, even in GaAs. In our own comparisons, when using 

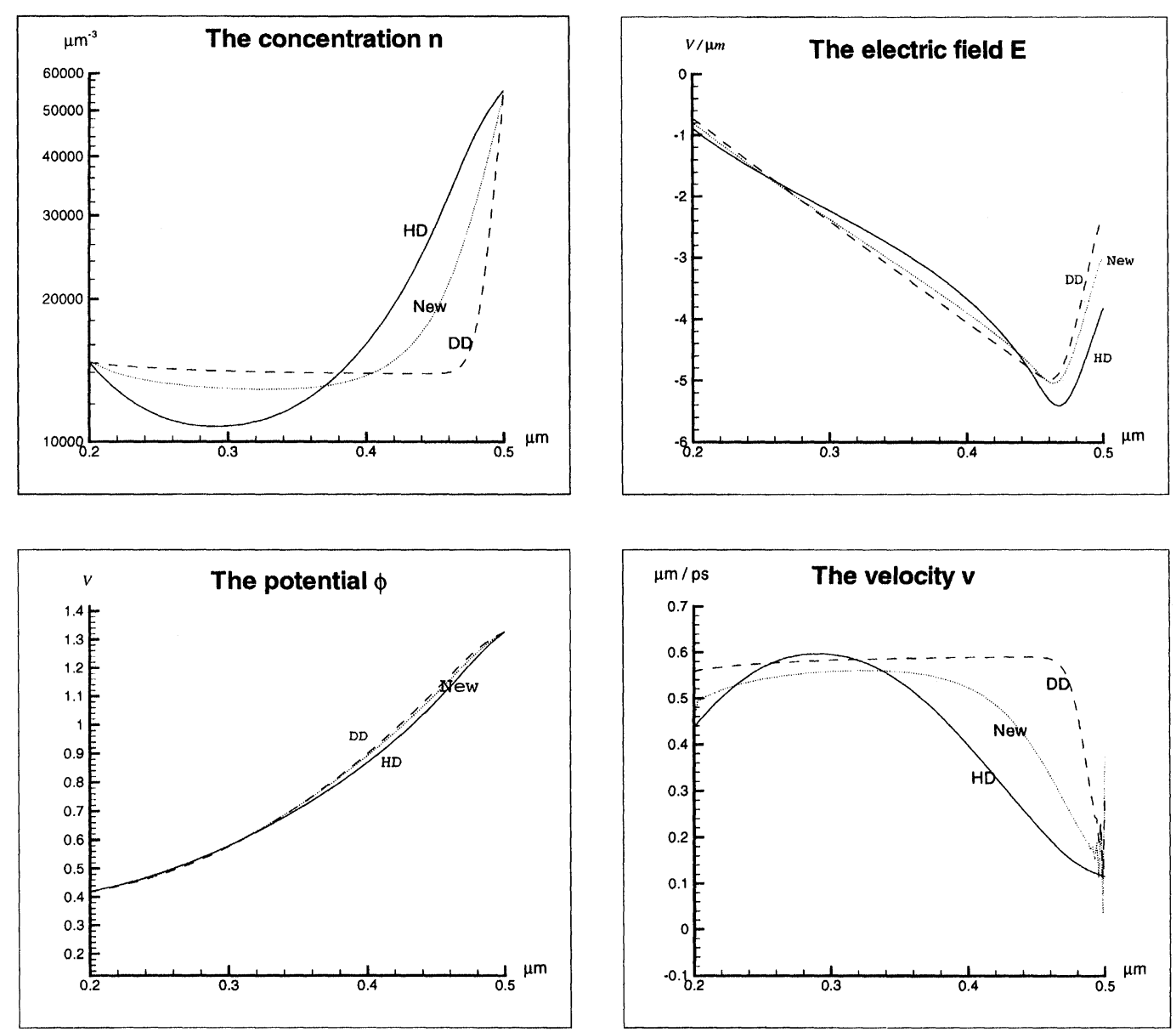

FIGURE 4 The comparison of the Drift-Diffusion (DD) model (dashed line), the new high field model (dotted line), and the HydroDynamic (HD) model (solid line), in the sub-interval $[0.2,0.5]$ using the HD results as boundary conditions. GaAs, $T_{0}=300$, $\nu$ bias $=1.0$. Top left: concentration $n$ in $\mu \mathrm{m}^{-3}$; top right: the electric field $E=-\phi_{x}$ in volts $/ \mu \mathrm{m}$; bottom left: the potential $\phi$ in volts; bottom right: the velocity $v$ in $\mu \mathrm{m} / \mathrm{ps}$.

derived velocity, and a field dependent mobility in $\mathrm{DD}$, we did not experience such a pronounced understatement. We have presented the results of the simulations in Figure 1 for comparison with standard DD, and in Figure 2 for comparison with the high field model. And, remarkably, we find that in the channel, as a problem posed only there, DD overstates the derived velocity! Moreover, the high field model predicts a velocity intermediate between the DD model and the hydrodynamic model.

Paper [1] is difficult to correlate directly because emphasis is placed upon frequency distributions. Nonetheless the average velocity and electric field calculations made there compare favorably with our own. Issues of heating and cooling discussed there are not raised in the present paper, but we intend to consider the effect of energetics in future work. The model has already been developed by the second author.

\section{ALGORITHM}

We shall only briefly describe the algorithm used in this paper, namely the ENO scheme developed in [8]. The ENO scheme is designed for a system of hyperbolic conservation laws of the form, 


$$
u_{t}=f(u)_{x}=g(u, x, t),
$$

where $u=\left(u_{1}, \ldots, u_{m}\right)^{T}$, and the hyperbolicity condition,

$$
\frac{\partial f}{\partial u} \text { is diagonalizable, with real eigenvalues, }
$$

holds. An initial condition is adjoined to (5.1).

For systems of conservation laws, local field by field decomposition is used, to resolve waves in different characteristic directions. For this purpose, analytical expressions are needed for the eigen-values and eigenvectors of the Jacobian matrix. This reduces the determination of the scheme to the case of a single conservation law. Thus, to describe the schemes, consider the scalar one dimensional problem, and a conservative approximation of the spatial operator given by

$$
L(u)_{j}=-\frac{1}{\Delta x}\left(\hat{f}_{j+\frac{1}{2}}-\hat{f}_{j-\frac{1}{2}}\right) .
$$

Here, the numerical flux $\hat{f}$ is assumed consistent:

$$
\hat{f}_{j+\frac{1}{2}}=\hat{f}\left(u_{j-1}, \ldots, u_{j+k}\right) ; \quad \hat{f}(u, \ldots, u)=f(u) .
$$

The conservative scheme (5.2), which characterizes the $\hat{f}$ divided difference as an approximation to $f(u)_{x}$, suggests that $\hat{f}$ can be identified with an appropriate function $h$ satisfying

$$
f(u(x))=\int_{x-\frac{\Delta x}{2}}^{x+\frac{\Delta x}{2}} h(\xi) d \xi .
$$

If $H$ is any primitive of $h$, then $h$ can be computed from $H^{\prime} . H$ itself can be approximated by polynomial interpolations using Newton's divided difference method, beginning with differences of order one, since the constant term is arbitrary. The necessary divided differences of $H$, of a given order, are expressed as constant multiples of those of $f$ of order one lower. The main ingredient of the ENO method is the adaptive choice of stencil: it begins with a starting point to the left or right of the current "cell" by means of upwinding, as determined by the sign of the derivative of a selected flux (or the eigenvalue of the Jacobian in the system case); as the order of the divided differences is increased, the divided differences themselves determine the stencil: the "smaller" divided difference is chosen from two possible choices at each stage, ensuring a smoothest fit.

\section{Acknowledgments}

The second author is supported by the National Science Foundation under grant DMS-9623037. The third author is supported by the National Science Foundation under grant DMS-9424464. The fourth author is supported by the National Science Foundation under grants ECS-9214488 and ECS-9627849, and the Army Research Office under grant DAAH04-94-G-0205. Computation is supported by the Pittsburgh Supercomputer Center.

\section{References}

[1] Barenger, H. U. and Wilkins, J. W. (1987). Ballistic structure in the electron distribution function of small semiconducting structures: General features and specific trends. Physical Review B, 36, 1487-1502.

[2] Cercignani, C., Gamba, I. M., Jerome, J. W. and Shu, C.W. Applicability of the high field model: An analytical study via asymptotic parameters defining domain decomposition, this volume.

[3] Cercignani, C., Gamba, I. M. and Levermore, C. L. (1997). High field approximations to a Boltzmann-Poisson system and boundary conditions in a semiconductor. Appl. Math. Lett., 10, 111-117.

[4] Jerome, J. W. (1996). Analysis of Charge Transport; A Mathematical Theory of Semiconductor Devices, Springer.

[5] Jerome, J. W. and Shu, C.-W. (1994). Energy models for one-carrier transport in semiconductor devices. In IMA Volumes in Mathematics and Its Applications, 59, Springer, $185-207$

[6] Jerome, J. W. and Shu, C.-W. (1995). Transport effects and characteristic modes in the modeling and simulation of submicron devices. IEEE Trans. CADICAS, CAD-14, 917-923.

[7] Kan, E. C., Ravaioli, U. and Kerkhoven, T. (1991). Calculation of velocity overshoot in submicron devices using an augmented drift-diffusion model, Solid-State Electr., 34, 995-999.

[8] Shu, C.-W. and Osher, S. J. (1989). Efficient implementation of essentially non-oscillatory shock capturing schemes, II. J. Comp. Physics, 83, 32-78.

[9] Thornber, K. K. (1983). Current equations for velocity overshoot, IEEE Electron Device Lett., 3, 69-71. 


\section{Authors' Biographies}

Carlo Cercignani received his Univ. Milano Laurea Physics in 1961 and his Univ. Milano Laurea Mathematics in 1963. From 1963 to 1966 he was Assistant and Associate Professor Of Plasma Physics at the University of Milano. He was a Visiting Associate Professor of Applied Mathematics at M.I.T. during 1966-67. He came to Politecnico di Milano in 1968 and has been Professor in Theoretical Mechanics since 1975. His scientific specialties include the kinetic theory of gases and its applications to rarefied gas dynamics, neutron transport and semiconductors; nonequilibrium statistical mechanics, fluid dynamics and singular integral equations. His current research interests are analytical and numerical treatment of kinetic and transport equations for gases and semiconductors. $\mathrm{He}$ is a member of Accademia Nazionale dei Lincei, Istituto Lombardo and Académie des Sciences de Paris. In addition he is a member of the National Commitees for Mathematics of the Italian Research Council (C.N.R., President), Scientific Committee of the National Group for Mathematical Physics (G.N.F.M.) of C.N.R., a member of General Assembly of IUTAM, General Assembly of IMU, Congress Committee of IUTAM, Scientific Committee of 1st European Mathematical Congress, and Advisory Committee for Symposia on Rarefied Gas Dynamics. He serves on the following editorial boards (1993): Fluid Dynamics Research Transport Theroy and Statistical Physics, MECHANICS Research Communications, Annali di Matematica Pura e Applicata, Applied Mathematics Letters, European Journal of Mechanics B (Ass. Editor), Nonlinear Differential Equations and Applications, Surveys in Mathematics for Industry, Continuum Mechanics and Thermodynamics. He has authored or coauthored seven scientific books and more than two hundred scientific publications. He is the recipient of the Gold Medal for Mathematics of the Accademia dei XL (1982), Prize "Citta' di Cagliari" for Applied Mathematics (1992), Docteur Honoris
Causa of the University Pierre et Marie Curie (Paris VI) (1992) and the Humboldt Prize (Academic Year 1995-1996).

Irene M. Gamba received the Ph.D. degree in Mathematics from The University of Chicago in 1989. From 1990 to 1991 she was at Purdue University as a Visiting Assistant Professor and Research Associate. She was an Assistant Professor at The College of New Jersey, Trenton, NJ, during 1991-92. She came to the Courant Institute of Mathematical Sciences, New York University, in 1992. There she was an NSF Postdoctoral Fellow during 1992-94, an Assistant Professor from 1994-96, and Associate Professor during 1996-97. She has accepted a position as Professor of Mathematics at the University of Texas at Austin beginning in Augest, 1997. Her research interests include nonlinear analysis applied to gas dynamics and charged-particle transport systems in the mathematical modeling of microelectronic devices.

Joseph W. Jerome received the Ph.D. degree in Mathematics from Purdue University in 1966. He was visiting Assistant Professor at the Mathematics Research Center, University of Wisconsin, during 1966-68, and was Assistant Professor at Case Western Reserve University during 1968-70. He joined Northwestern University in 1970, where he has been Professor of Mathematics and Applied Mathematics since 1976. He has held sabbatical positions at Oxford University, England, 197475, University of Texas, 1978-79, and Bell Laboratories, Murray Hill, 1982-83. He was visiting scholar at the University of Chicago in 1985. He received the Distinguished Alumnus Award from Purdue University's School of Science in 1996. His research interests include applied analysis, numerical analysis, computational electronics, and ion transport in biology. The most recent of his three books, Analysis of Charge Transport, was published by Springer in 1996.

Chi-Wang Shu received the B.S. degree in Mathematics from the University of Science and Technology of China (USTC) in 1982 and the Ph.D. degree in Applied Mathematics from the University 
of California at Los Angeles (UCLA) in 1986. He was a postdoctoral fellow at the the Institute for Mathematics and Its Applications (IMA), University of Minnesota, during 1986-1987, and came to Brown Uinversity in 1987, where he has been Professor of Applied Mathematics since 1996. He was a co-recipient of the Chinese Academy of
Science Award in Numerical Analysis and Scientific Computing in 1995. His research interests include numerical analysis, scientific computing and computational physics. He currently serves on the editorial boards of Mathematics of Computation, SIAM Journal on Numerical Analysis, and Journal of computational Mathematics. 

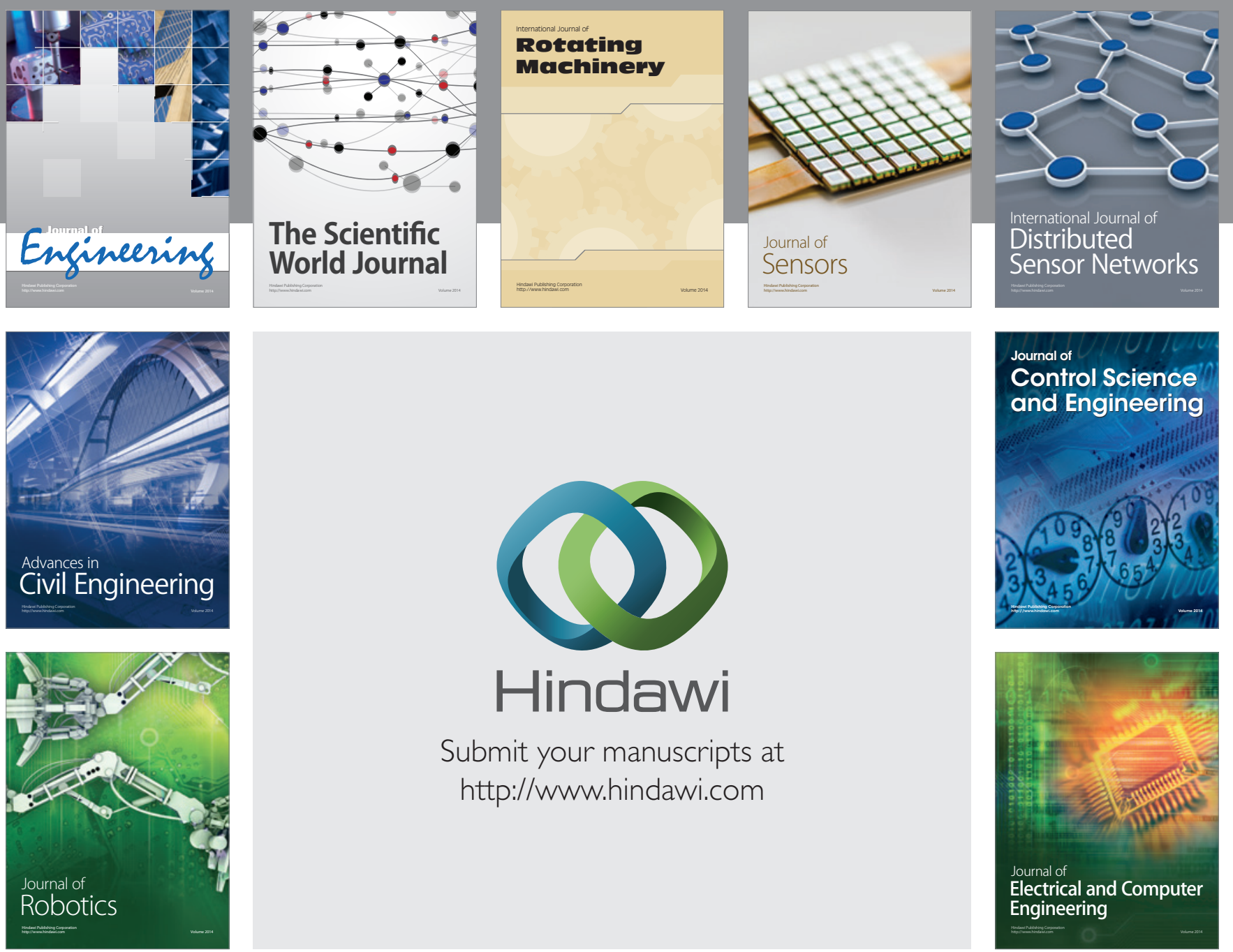

Submit your manuscripts at

http://www.hindawi.com
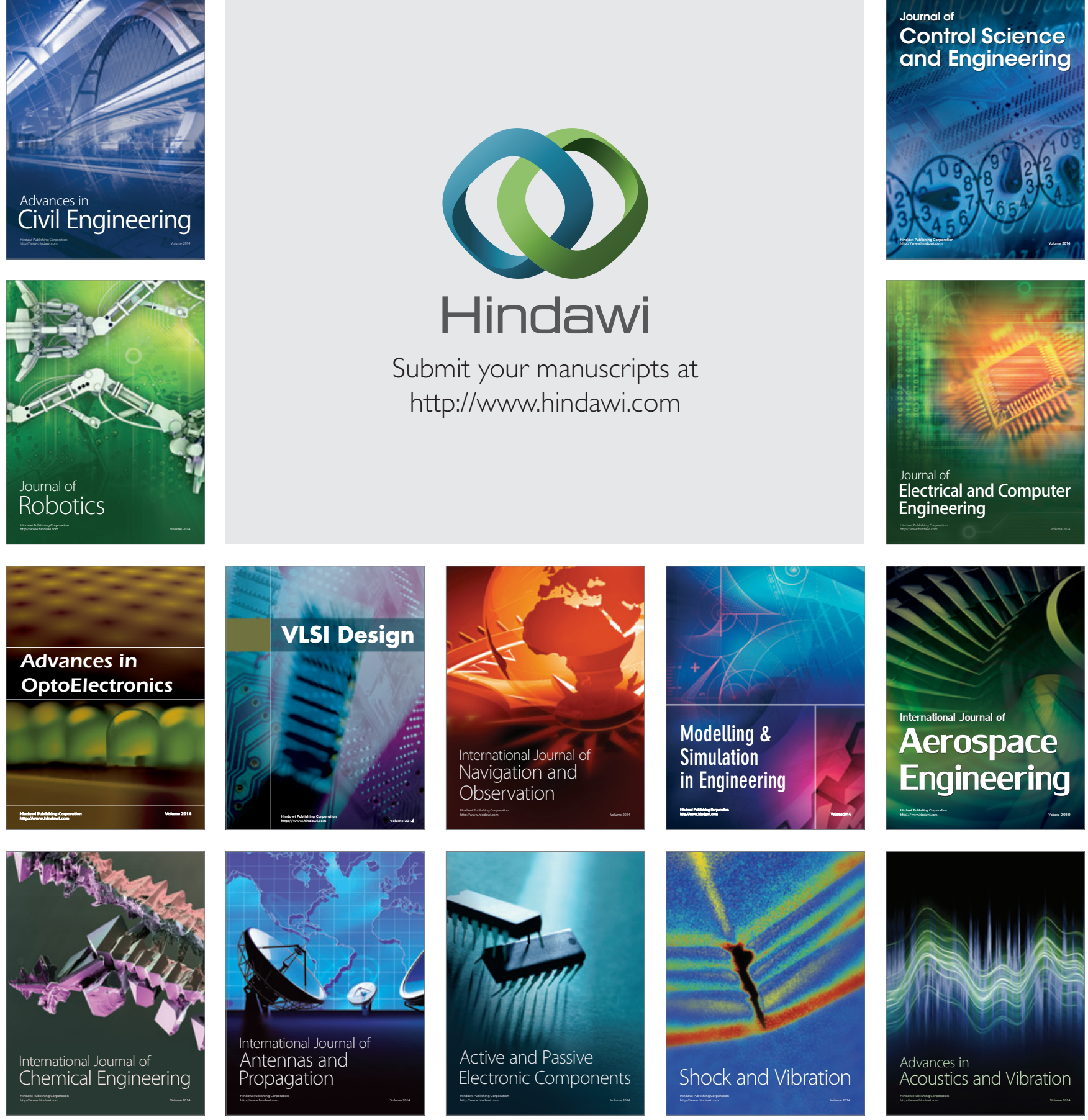